\title{
Empowerment in der agilen Arbeitswelt
}

\section{ein Schlüssel für die nachhaltige Gestaltung neuer Arbeitsformen}

\author{
Andreas Boes, Katrin Gül, Tobias Kämpf@ und Thomas Lühr
}

\subsection{Das Projekt „Empowerment in der digitalen Arbeitswelt“}

In der digitalen Transformation stehen etablierte Unternehmen vor der Herausforderung, sich in einem umfassenden Sinn neu zu erfinden. Dies reicht von der Entwicklung neuer Geschäftsmodelle über das Neudenken von Innovationsstrategien und Produktionsprozessen bis hin zur Einführung neuer Organisationsformen von Arbeit. In diesem Neuorientierungsprozess erleben wir, wie gerade in den Vorreiter-Unternehmen des Umbruchs die Frage nach der Rolle der Mitarbeitenden und Führungskräfte neu gestellt wird. Ob dies in der Folge zu einer Neugestaltung der Arbeitswelt im Sinne der Menschen führen wird oder nicht, wird allerdings entscheidend davon abhängen, ob es gelingt, die Beschäftigten konsequent zu empowern. Daher ist das konsequente Empowerment der Beschäftigten der Schlüssel für den langfristigen Erfolg agiler und humaner Arbeitswelten und muss in der Unternehmenspraxis einen zentralen Stellenwert einnehmen.

Ziel des Verbundprojekts „Empowerment in der digitalen Arbeitswelt“ (EdA) ${ }^{1}$ war es daher, zusammen mit Vorreiterunternehmen Konzepte für ein Empowerment

\footnotetext{
${ }^{1}$ Das Projekt „Empowerment in der digitalen Arbeitswelt“ (EdA) ist ein Verbundprojekt unter der Leitung des Instituts für Sozialwissenschaftliche Forschung e. V. - ISF München und in Zusammenarbeit mit der Universität Kassel, dem Betriebsrat der AUDI AG Ingolstadt, der IG Metall und der andrena objects ag (Laufzeit: 1. Januar 2017 bis 30. April 2020). Es wird durch Mittel des Bundesministeriums für Bildung und Forschung (BMBF) im Programm „Innovationen für die Produktion, Dienstleistung und Arbeit von morgen“" und des Europäischen Sozialfonds (ESF) gefördert und vom Projektträger Karlsruhe (PTKA) betreut.
}

A. Boes $\cdot$ K. Gül · T. Kämpf $(\bowtie) \cdot$ T. Lühr ISF München e. V., München, Deutschland 
von Beschäftigten zu entwickeln, die eine nachhaltige Gestaltung der agilen Arbeitswelt ermöglichen. Sieben zentrale Gestaltungsfelder standen dabei im Fokus: „Agile Organisationskonzepte“, „neue Führungskulturen“, „Gesundheitsförderung“, „Zeitsouveränität“", ,Team-Empowerment“, „Partizipation \& Mitbestimmung“ und „Crowd“.

\subsection{Empowerment: Wie kann die neue Arbeitswelt nachhaltig gestaltet werden?}

Die Grundlage für den aktuellen Umbruch in Wirtschaft und Gesellschaft bildet der Aufstieg des Internet und seine Entwicklung zu einem global verfügbaren „Informationsraum“ [2, 3]. Dieser Informationsraum wird zum Ausgangspunkt für die Herausbildung einer neuen Form von Ökonomie, der „Informationsökonomie“ [9: 122]. In der Informationsökonomie bildet das Konzept der Cloud die Basis für eine grundlegende Neuorganisation der Geschäftsmodelle, der Wertschöpfung sowie der Innovations- und Arbeitsprozesse. Die Cloud wird damit zum Wegbereiter eines regelrechten Paradigmenwechsels in den Unternehmen (vgl. ebd.; [21]). Komplementär zur Entwicklung digitaler Geschäftsmodelle im Informationsraum geht es dabei auf organisatorischer Ebene insbesondere um die kurzzyklische Auslieferung von Innovationen, um das Aufbrechen von starren Abteilungsgrenzen, um ,flache Hierarchien“, um mehr Flexibilität und um Kundenorientierung. Im Zuge dieses Paradigmenwechsels entstehen umfangreiche neue Möglichkeiten, welche die Unternehmen für ihre Produkt- und Geschäftsstrategien wie auch für die Arbeitsorganisation nutzen können [9].

\subsubsection{Agilität: Die neue Leitorientierung}

Um diese neuen Möglichkeiten der Digitalisierung nutzen und letztlich auch bewältigen zu können, suchen neben Start-ups auch etablierte Unternehmen seit Jahren nach einem neuen Bauplan [6, 7]. Wir konnten im Zuge unserer empirischen Untersuchungen feststellen, dass sich in diesem Prozess der Neuerfindung vor allem das Konzept der Agilität als eine neue Leitorientierung durchzusetzen beginnt. Dabei wird insbesondere die traditionelle Managementkultur des ,command \& control“ infrage gestellt. Stattdessen gewinnen Ansätze, die auf Eigenverantwortung und Selbstorganisation von Beschäftigten basieren, an Bedeutung.

In dieser Phase der Neuerfindung experimentieren die Unternehmen mit vielfältigen agilen Konzepten und Methoden. Beispiele hierfür sind etwa die Einführung agiler Methoden, wie Scrum oder Kanban [4, 18], die Nutzung Community-basierter Ansätze von Wissenstransfer oder verschiedenste Formen eines agilen „Staffings“, z. B. Crowdsourcing [33] bzw. ,interne Crowd Work“ (vgl. [17, 27]), bis hin zu einer Schwarmorganisation. Gemeinsam ist diesen unterschiedlichen Ansätzen vor allem eines: Sie sind Ausdruck einer Suche nach Alternativen zu bürokratischen Organisationskonzepten. 
Mit diesen Bestrebungen, nicht nur die Organisationsstrukturen, sondern auch die Prozesse und die Arbeitsorganisation agiler zu gestalten, sind - zumindest in den Vorreiter-Unternehmen - auch ganz neue Vorstellungen davon verbunden, wie sich Beschäftigte in den Arbeitsprozess einbringen sollen. Dahinter steht die Überlegung, dass sich die vielfältigen und in rasanter Entwicklung begriffenen Möglichkeiten der digitalen Transformation nur dann in einer angemessenen Geschwindigkeit nutzen lassen, wenn sich die Beschäftigten sich in ihren Verantwortungsbereichen mit ihren Kompetenzen, ihrem Know-how und ihrer Einsatzbereitschaft reaktionsschnell dazu ins Verhältnis setzen können. Dabei lassen sich verschiedene Tendenzen beobachten, welche am bisherigen Rollenverständnis von Mitarbeitenden und Führungskräften rütteln (vgl. [8]).

So sollen die Mitarbeitenden lernen, eigenverantwortlich $\mathrm{zu}$ agieren und nicht mehr lediglich auf Vorgaben und exakte Anweisungen von Vorgesetzten zu reagieren. Umgekehrt wird an Führungskräfte die Anforderung gestellt, „loszulassen“ und als „Enabler“ für das Empowerment und die Selbstorganisation des Teams zu fungieren. Ausgehend von der Annahme, dass die qualifizierten Mitarbeitenden selbst am besten wissen, wie ihre Arbeit funktioniert, sollen sie diese auch selbst organisieren und planen. Die bestehenden bürokratischen Prozesse sollen von den Mitarbeitenden hinterfragt und in ,intelligente Prozesse“ überführt werden, die immer wieder neu an die jeweils gegebenen Anforderungen angepasst werden können.

Doch was bedeuten all diese Veränderungstendenzen für die Beschäftigten in den Unternehmen? Führen sie tatsächlich zu einem Autonomiezuwachs und zu mehr Mitbestimmungsmöglichkeiten aufseiten der Beschäftigten? Können sie ihre Arbeitsabläufe selbstbestimmt gestalten und haben Zugang zu den benötigten und gewünschten Informationen? Kurzum: Führen die neuen Anforderungen an Beschäftigte in agilen Arbeitsumgebungen tatsächlich zu einem echten Empowerment? Kritische Stimmen sehen in den Bestrebungen, die Selbstorganisation der Beschäftigten zu stärken, vor allem den Versuch, bisher ungenutzte Potenziale für die Bewältigung der neuartigen Herausforderungen im Zuge der digitalen Transformation nutzbar zu machen und so eine Effizienzsteigerung zu erzielen (vgl. z. B. [11]). Eng mit dieser Entwicklung verbunden ist aber auch eine Neuauflage der Diskussion um neue Chancen für Partizipation und Selbstbestimmung im Sinne einer Humanisierung von Arbeit [7].

Schon einmal, im Zuge der Einführung „,neuer Produktionskonzepte“ [20] nach dem krisenhaften Umbruch in den 1970er und 1980er Jahren, erhielten Formen direkter Partizipation und Mitarbeiterbeteiligung Auftrieb (ausführlich: Boes et al. 2018). Schon damals wurde die Idee einer Vereinbarkeit von Humanisierung und Rationalisierung kritisch diskutiert $[15,16,26]$. Und auch damals stand die Nutzung der Produktionsintelligenz der Beschäftigten im Mittelpunkt: Eine Erweiterung der Entscheidungskompetenzen der ausführenden Ebene geschah mit der Idee, wichtige Produktivitätspotenziale durch die Förderung der fachlichen Souveränität der Arbeiter zu erschließen. Ähnliches galt später auch für den Ansatz der Lean Production (z. B. [24]). Insgesamt konnte die Umsetzung von Formen direkter Partizipation und beteiligungsorientierter Managementprinzipien in den bisherigen Suchprozessen nach Alternativen 
zum fordistisch-bürokratischen Unternehmensmodell jedoch kaum das Anfangsstadium überschreiten (z. B. [14-16]).

\subsubsection{Ein neuer Möglichkeitsraum für mehr Empowerment}

Mit dem digitalen Umbruch eröffnet sich nun ein neuer Möglichkeitsraum für mehr Demokratie in der Arbeitswelt und eine Beteiligung der Beschäftigten. Wir erleben gegenwärtig, wie der gesamte Bauplan des fordistisch-bürokratischen Unternehmens grundlegend zur Disposition gestellt wird - von der Abschottung funktionaler Säulen über das hierarchische Führungsverständnis bis hin zum Expertenmodus hochqualifizierter Kopfarbeit [5]. In den Unternehmen gewinnt damit ein umfassender Neuerfindungsprozess an Dynamik, welcher mit einem neuen Rollenverständnis von Beschäftigten und Führungskräften einhergeht und ein Empowerment der Beschäftigten ermöglichen kann.

Wir können also festhalten, dass aus den neuen Anforderungen an die Beschäftigten im Zuge der Verbreitung agiler Arbeitsformen allein noch kein Empowerment der Beschäftigten erwächst. Vielmehr entsteht ein neuer Möglichkeitsraum, um Strukturen $\mathrm{zu}$ schaffen, die ein konsequentes Empowerment von Beschäftigten ermöglichen. Dabei haben uns unsere Forschungsergebnisse ${ }^{2}$ deutlich gezeigt: Empowerment ist die Voraussetzung dafür, dass agile Methoden nicht nur formal umgesetzt, sondern auch wirklich gelebt werden (vgl. [35]). Erst ein konsequentes Empowerment gibt den Beschäftigten die Möglichkeit, ihre Arbeit so zu gestalten, dass sie zielorientiert, sinnerfüllend und nachhaltig zugleich ist. Nur wenn Empowerment gelebt wird, können Teams gemeinsam Arbeitsprozesse so gestalten, dass sie in einem angemessenen Zeitrahmen $\mathrm{zu}$ erfüllen sind und jedes Teammitglied die Unterstützung bekommt, die es braucht. Damit ist Empowerment auch die Grundlage dafür, dass Beschäftigte bereit sind, ihr Wissen zu teilen, und dass Lernprozesse im Team wie im Unternehmen stattfinden können. Das Empowerment der Beschäftigten ist eine Voraussetzung dafür, negativen Beanspruchungen in agilen Arbeitsformen vorzubeugen. Empowerment ist der Schlüssel für ein nachhaltiges agiles Arbeiten.

\subsubsection{Empowerment: strukturell und psychologisch}

Eine präzise Begriffsbestimmung von Empowerment ist nicht ganz einfach, da das Konzept seit seinem Entstehen in sehr heterogenen Bereichen mit teilweise unterschiedlichen politischen Intentionen aufgegriffen wurde (vgl. [11]).

\footnotetext{
${ }^{2}$ Im Rahmen des Teilvorhabens des ISF München wurden insgesamt 86 Expertengespräche sowie Beschäftigteninterviews in acht verschiedenen Unternehmen aus der IT-, Automobil-, Elektrosowie Energiebranche geführt.
} 
Die Ursprünge des Konzepts reichen in die 70er Jahre des 20. Jahrhunderts zurück, als es von der amerikanischen Bürgerrechtsbewegung geprägt und anschließend vor allem von der gemeindebezogenen Sozialen Arbeit aufgegriffen wurde, insbesondere bei Julian Rappaport ${ }^{3}$ (vgl. [23]). Im Mittelpunkt dieses Ansatzes steht eine ressourcenorientierte Perspektive, welche nicht die „Mängel“" von Menschen in den Blick nehmen möchte, sondern vielmehr auf die Stärkung von Potenzialen abzielt.

Schon früh wurde das Empowerment-Konzept von der betrieblichen Managementforschung adaptiert. In der Managementlehre werden häufig zwei unterschiedliche Ansätze von Empowerment gegenübergestellt (vgl. [10: 861 f.] oder [34]): das strukturelle Empowerment und das psychologische Empowerment. Während das strukturelle Empowerment mit einer Makro-Perspektive assoziiert wird, da hierbei Strukturen, Strategien und Praktiken in den Blick genommen werden, stellt das psychologische Empowerment eine Mikro-Perspektive auf die Wahrnehmungen der Mitarbeitenden dar (vgl. [28]).

Das strukturelle Empowerment geht auf Rosabeth Moss Kanter [19] zurück. Es fokussiert darauf, Entscheidungsmacht an niedrigere Hierarchieebenen $\mathrm{zu}$ delegieren, um auf diese Art bessere Arbeitsresultate zu erhalten und die Produktivität des Unternehmens zu erhöhen. Dabei benennt [19] fünf Bedingungen in der Arbeit, welche Voraussetzung für das Empowerment der Beschäftigten sind:

- Möglichkeit, sich selbst weiterzuentwickeln und zu wachsen

- Zugang zu relevantem Wissen

- Zugang zu Unterstützungsleistungen (Feedback/Beratung durch Kollegen bzw. Vorgesetzte)

- Zugang zu adäquaten Ressourcen (Zeit, Mittel)

- Gelegenheit zum Aufbau und zur Nutzung von persönlichen Netzwerken

Auch wenn Kanter damit wichtige Faktoren von Empowerment hervorhob, galt die isolierte Perspektive auf ein strukturelles Empowerment als unvollständig. So merkten [13: 474] an, dass eine alleinige Perspektive auf diese Seite des Empowerments die Selbstwirksamkeit der Mitarbeitenden unberücksichtigt lässt, und Spreitzer [29] vermisste die Erfassung der Wahrnehmung von bestimmten Strukturen und Praktiken durch die Mitarbeitenden. Sie entwickelte daraufhin das in der Empowerment-Forschung fest etablierte und vielfach validierte Konzept des psychologischen Empowerments (vgl. [1, 12, 22]).

Im Gegensatz zum strukturellen Empowerment steht beim psychologischen Empowerment weniger die tatsächliche Weitergabe von Autorität und Verantwortung im Mittelpunkt, sondern vielmehr die subjektive Wahrnehmung empowernder Arbeits-

\footnotetext{
${ }^{3}$ Julian Rappaport [25] machte das Konzept bekannt. In seinem maßgeblichen Artikel von 1981 hieß es: „Having rights but no resources and no services available is a cruel joke.“
} 
bedingungen durch die Mitarbeitenden und deren damit verbundene kognitive Zustände $[13,29]$. Die dahinterliegende Annahme ist, dass das Ausmaß, in dem die Organisation Empowerment-Maßnahmen implementiert, und das Ausmaß, in welchem sich Mitarbeiter empowert fühlen, nicht zwangsläufig übereinstimmen [31]. Psychologisches Empowerment setzt sich nach Spreitzer aus vier Wahrnehmungen zusammen: dem Empfinden von Bedeutsamkeit, dem Empfinden von Kompetenz, der Erfahrung von Selbstwirksamkeit sowie dem Erleben von Einflussnahme [30]. In ihrer Gesamtheit gelten diese Wahrnehmungen als motivierendes Element für eine proaktive Haltung in der Arbeit [32].

\subsubsection{Empowerment: Ganzheitlich gedacht}

In der Empowerment-Literatur wurde jedoch - nicht zuletzt durch Spreitzer selbst immer wieder darauf verwiesen, dass beide Perspektiven auf Empowerment - also die strukturelle ebenso wie die psychologische - im Zusammenspiel zu betrachten sind (z.B: $[30,31])$.

Dieser Auffassung schließen wir uns an. Wir begreifen Empowerment als ein gelingendes Wechselverhältnis zwischen den betrieblichen Rahmenbedingungen, welche das aktive Engagement der Menschen ermöglichen und fördern, und der Bereitschaft der Menschen, sich entsprechend in das Unternehmen einzubringen. Wir halten es allerdings für notwendig, noch einen Schritt weiter zu gehen: Die genannten Empowerment-Konzepte, die ihren Ursprung zum Teil schon in den 70er Jahren des 20. Jahrhunderts haben, denken Empowerment immer noch als Bestandteil einer traditionell hierarchischen Struktur. Empowerment wird dann meist mit der Delegation von Verantwortung und dem Zugestehen von Entscheidungs- und Handlungsspielräumen gleichgesetzt (vgl. Bröckling 2003). Im Zuge unserer empirischen Erhebungen haben wir allerdings gelernt, dass das Empowerment der Beschäftigten von ganz unterschiedlichen Aspekten in den Unternehmen bestimmt wird. Der digitale Umbruch und die agile Leitorientierung in den Unternehmen führen dazu, dass das Empowerment der Beschäftigten auf vielfältigen Ebenen an Bedeutung gewinnt und hier konkret wird. Wir möchten daher im Folgenden die inhaltlichen Dimensionen von Empowerment in den Unternehmen bestimmen und damit zu einem ganzheitlichen und systematischen Verständnis von Empowerment in der digitalen Transformation beitragen.

\subsubsection{Empowerment in der agilen Arbeitswelt: die zentralen Dimensionen}

Angesichts der Tatsache, dass sich aktuell in vielen Unternehmen ein neuer Möglichkeitsraum öffnet, um die Weichen für ein nachhaltiges Empowerment im Sinne der Menschen und der Unternehmen zu stellen, halten wir es für notwendig, ein genaueres Verständnis davon zu haben, worin das Empowerment der Menschen in den Unter- 


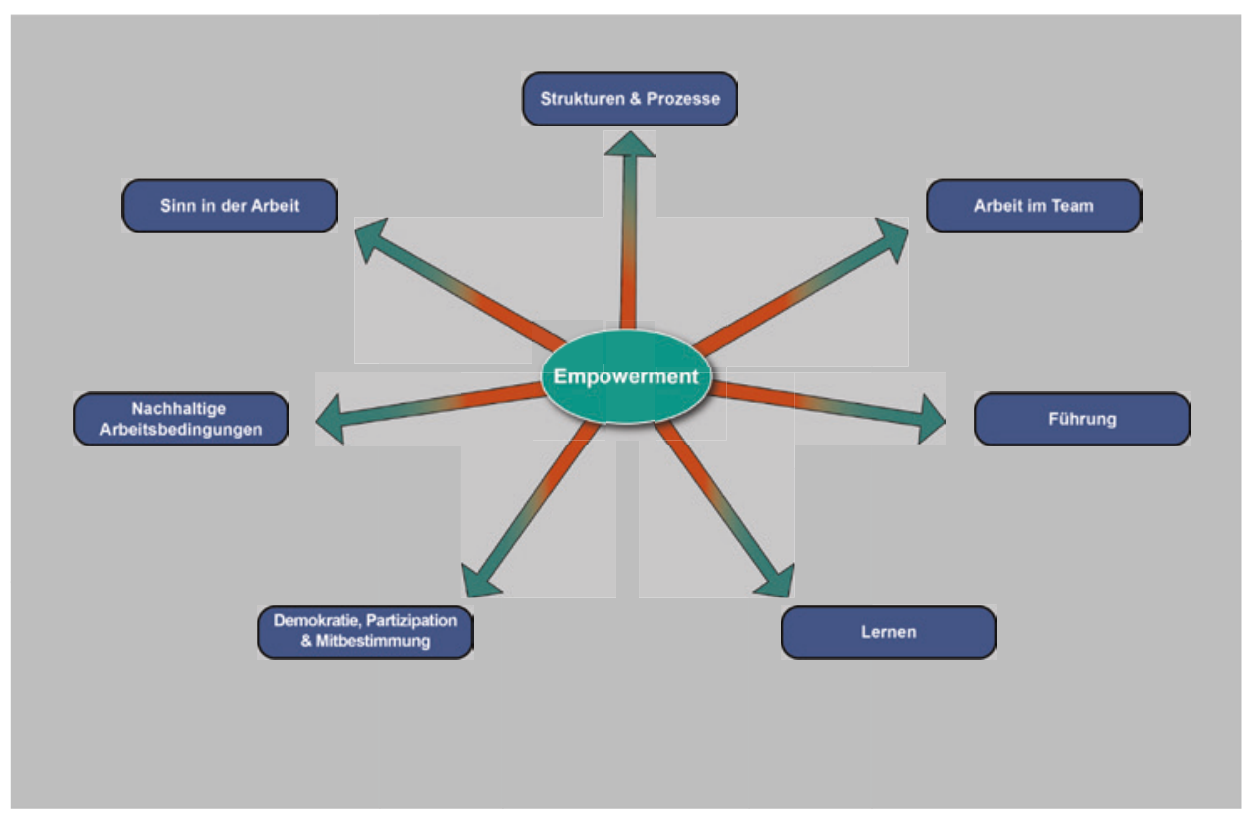

Abb. 20.1 Dimensionen von Empowerment in der agilen Arbeitswelt, eigene Abbildung

nehmen konkret besteht. Die folgenden sieben Dimensionen von Empowerment halten wir dabei für zentral (Abb. 20.1):

Führung: Die Organisation von Führung ist entscheidend bei der Frage, ob es gelingt, das Empowerment der Beschäftigten in den Unternehmen systematisch zu ermöglichen und zu fördern. In agilen Organisationen bedeutet das, dass Führung zu einer gemeinschaftlichen Aufgabe werden muss. Anstelle von hierarchischer Anweisung durch Einzelne wird ein Konzept von Führung benötigt, das auf sozialen Aushandlungsprozessen basiert. Dies betrifft Aushandlungsprozesse im Team ebenso wie zwischen verschiedenen funktionalen Rollen, die jeweils unterschiedliche Perspektiven auf das Ganze darstellen. Eng damit verbunden ist auch die Bereitschaft des Managements, die Autonomie agiler Teams zu akzeptieren. Das Führen empowerter Mitarbeiter bedeutet daher auch, dass sich die Rolle der Führungskraft grundlegend verändert: Statt Kontrolle gewinnen Unterstützung und Beratung als Funktionen von Führung an Bedeutung. Dies beinhaltet beispielsweise die Unterstützung beim Zugang zu wichtigen Ressourcen wie Informationen und notwendigem Know-how, beim Erwerb von Qualifikationen oder auch bei der Realisierung von Finanz- und Zeitplänen.

Strukturen und Prozesse: Für das Empowerment der Mitarbeiter ist es entscheidend, dass sie im Unternehmen Strukturen und Prozesse vorfinden, welche eine flexible Anpassung an sich verändernde Ansprüche und Erfordernisse sowie einen offenen und leichten Austausch über Abteilungsgrenzen hinweg ermöglichen. Hierbei ist wesentlich, 
inwieweit die Beschäftigten eine Organisationsstruktur erleben, die sie in ihrer Eigeninitiative befördert und nicht durch starre und bürokratische Prozesse behindert. Dazu gehören auch ein offener Umgang mit Informationen, die Möglichkeit, neue Arbeitsformen auszuprobieren, sowie das Angebot von Plattformen und Tools, die eine weitgehend barrierefreie Zusammenarbeit ermöglichen.

Arbeit im Team: Ein empowertes Team verfügt über Entscheidungsfreiräume, Arbeitsabläufe selbstbestimmt zu gestalten und eine kollektive Strategie- und Handlungsfähigkeit zu entwickeln. Diese kann es zum einen zur Erschließung von Sinnpotenzialen und persönlicher Entfaltung nutzen und zum anderen zur Grundlage für die Steuerung der eigenen Arbeitsmenge sowie für einen schonenden Umgang mit der eigenen Arbeitskraft machen. Für das Empowerment der Teammitglieder entscheidend ist, ob sich solide und ausgeprägte Vertrauensbeziehungen ausbilden können, die einen konstruktiven Umgang mit Transparenz ermöglichen. Anderenfalls können sich Formen eines Gruppen- und Rechtfertigungsdrucks entwickeln, die letztlich auch zur Entstehung neuer Belastungen führen können.

Lernen: Für das Empowerment der Mitarbeitenden ist der Umgang mit dem Thema „Lernen“ im Unternehmen essenziell. Dies geht weit über das Angebot von und den Zugang zu Weiterbildungs- und Qualifizierungsmaßnahmen hinaus. Es betrifft sehr viel umfassender die Frage, inwieweit es dem Unternehmen gelingt, durch die Etablierung kontinuierlicher Lernschleifen zu einer ,intelligenten Organisation“ zu werden. Dazu gehört beispielsweise ein konstruktiver Umgang mit Fehlern und Kritik oder auch die Etablierung von Freiräumen für Kreativität und Innovation. Eine wichtige Rolle spielt in diesem Kontext auch die Fähigkeit der Organisation, Erfahrungen und Verbesserungsvorschläge von Mitarbeitenden aufzunehmen und umzusetzen. Und auch das „Lernen voneinander" ist dabei ein wichtiges Thema: Es geht um die Frage, inwieweit es gelingt, im Unternehmen eine Vertrauenskultur zu erzeugen, die einen offenen Erfahrungs- und Know-how-Transfer zwischen den Mitarbeitenden ermöglicht und aktiv unterstützt.

Demokratie, Partizipation und Mitbestimmung: In welchem Maße partizipieren Beschäftigte an Unternehmensentscheidungen? Welche Möglichkeiten haben sie, selbst strategische Themen zu setzen und voranzutreiben? In welchem Umfang gibt es im Unternehmen kollektive Vereinbarungen, die das Empowerment der Beschäftigten nachhaltig sichern und das Vertrauen in neue Arbeitsformen stärken? Ein wichtiger Erfolgsfaktor für ein Empowerment der Beschäftigten ist eine beteiligungsorientierte Unternehmenskultur, die Selbstbestimmung durch Mitbestimmung ermöglicht. Gerade die institutionelle Absicherung der neuen Beteiligungsmöglichkeiten agiler Teams kann hier eine wichtige Grundlage schaffen. Dadurch ließe sich verhindern, dass z. B. die Dimensionen des Empowerments immer wieder zur Disposition gestellt werden und von den Teams neu verhandelt werden müssen. Stattdessen könnten die Dimensionen 
des Empowerments über Vereinbarungen zwischen den Sozialparteien verstetigt und den Beschäftigten ein verbriefter Anspruch auf Empowerment gewährt werden.

Nachhaltige Arbeitsbedingungen: Die Möglichkeiten für zeit- und ortsflexible Arbeitsformen wie mobiles Arbeiten oder Home-Office haben mit der Digitalisierung zugenommen. Sie können Beschäftigte wie Führungskräfte prinzipiell in die Lage versetzen, eine bessere Vereinbarkeit von Arbeits- und Privatleben zu erreichen. In der Praxis entscheidet allerdings das Empowerment der Beschäftigten bzw. das Ausmaß ihrer Zeitsouveränität darüber, ob die Flexibilisierung von Arbeitszeit und Arbeitsort der Realisierung nachhaltiger Arbeitsbedingungen dient oder zu einer Verlängerung der Arbeitszeiten sowie ausufernden Verfügbarkeitserwartungen führt. Entscheidend ist hierbei auch die Frage, inwieweit Beschäftigte die Anforderungen der Arbeit mit ihren sich wandelnden Bedürfnissen in unterschiedlichen Lebensphasen vereinbaren können.

Sinn in der Arbeit: Eine zentrale Komponente des psychologischen Empowerments ist das Empfinden von Bedeutsamkeit in der Arbeit. Hierbei ist es entscheidend, in welchem Umfang es eine Übereinstimmung zwischen den Zielen in der Arbeit und den persönlichen Einstellungen und Wertvorstellungen gibt und ob die Beschäftigten Arbeitsbedingungen vorfinden, in denen sie ihren eigenen Ansprüchen an Inhalt und Qualität in der Arbeit gerecht werden können. Auch die Frage, ob Beschäftigte hierbei das Gefühl der „Handhabbarkeit“ erfahren, also die Überzeugung, den Anforderungen gerecht werden zu können bzw. im Unternehmen etwas bewegen zu können, spielt bei der Erfahrung von Sinn eine wichtige Rolle.

Ein wichtiges Ergebnis und Produkt Verbundprojekts ist der Empowerment Index als ein integriertes Analyse- und Gestaltungstool. Auf Grundlage der identifizierten Dimensionen von Empowerment wurde ein ganzheitlich konzipiertes Analysetool zur Erfassung von Empowerment in der agilen Arbeitswelt entwickelt. Der EmpowermentIndex unterscheidet sich von anderen Instrumenten zur Erfassung von Empowerment, indem er in eine Theorie des digitalen Umbruchs eingebettet ist und zentrale Aspekte wie Führung sowie Demokratie und Mitbestimmung mit einbezieht. Das Tool soll die einzelnen Unternehmen dabei unterstützen, vor Ort mit den Beschäftigten und der Interessenvertretung spezifische Stärken, Schwächen und Bedarfe zu analysieren.

Der Index kann zum einen als reines Analysetool in einzelnen Abteilungen, aber auch in größeren Unternehmensbereichen zum Einsatz kommen. In diesem Fall gibt er Aufschluss darüber, wie die Beschäftigten ihre Gestaltungsspielräume in verschiedenen zentralen Bereichen der Arbeit wahrnehmen. Er eignet sich auch für ein kontinuierliches Monitoring. Zum anderen kann er als Arbeitsgrundlage für eine beteiligungsorientierte Gestaltung von Empowerment in den Organisationen dienen. Hierbei wird in Workshops mit einzelnen Abteilungen entlang der zentralen Empowerment-Dimensionen und gemeinsam mit den Beschäftigten der Ist- und Soll-Zustand bestimmt. Davon ausgehend werden Handlungsfelder für die Stärkung von Empowerment identifiziert und mögliche Gestaltungsansätze diskutiert. 


\subsubsection{Empowerment: Der humanistische Gegenentwurf zum digitalen Fließband}

Unter dem Eindruck des digitalen Umbruchs beginnt sich die agile Organisation als neue Leitorientierung in den Unternehmen durchzusetzen. Das eröffnet neue Chancen dafür, der Bedeutung des Menschen in der Digitalisierung gerecht zu werden und ihn in den Mittelpunkt zu stellen. Der Schlüssel dafür, damit das gelingen kann, ist die Frage des Empowerments: Nur wenn es gelingt, den Menschen im Arbeitsprozess zu empowern, kann er seiner neuen Rolle in der agilen Organisation gerecht werden. Die Bedeutung des Empowerments selbst geht allerdings weit über die Frage des Gelingens der agilen Organisation hinaus. Das Konzept des Empowerments markiert vor allem einen humanistischen Gegenentwurf zum Bedrohungsszenario der Digitalisierung als einer Intensivierung von Arbeit und Belastung an digitalen Fließbändern, als Vernichter von Arbeitsplätzen und einer sicheren Zukunftsperspektive sowie als Beschleuniger von Überwachung und Kontrolle in Arbeit und Gesellschaft. Dagegen steht Empowerment für die Perspektive eines Aufbruchs in eine neue Humanisierung der Arbeitswelt, in der die Möglichkeiten der Digitalisierung für die Menschen genutzt werden - und nicht gegen sie!

\subsection{Ausblick}

Immer mehr Unternehmen erkennen, dass die Rolle der Mitarbeitenden im Zuge der digitalen Transformation in den Unternehmen neu gedacht werden muss und dass dabei das Empowerment der Beschäftigten eine entscheidende Rolle spielt. Um diese Herausforderung nachhaltig bewältigen zu können, bedarf es eines ganzheitlichen Blicks auf das Empowerment unter den Bedingungen agilen Arbeitens. Hierzu liefern die Ergebnisse des Projekts EdA wichtige Erkenntnisse, die es bisher so noch nicht gibt.

Aufbauend auf diesen Ergebnissen ergeben sich zugleich eine Vielzahl neuer Forschungsfragen. So stellt sich vor dem Hintergrund fluider werdender Innen-AußenGrenzen der Unternehmen die Frage, wie Empowerment jenseits klassischer betrieblicher Strukturen gesichert werden kann und welche Konsequenzen sich daraus für eine nachhaltige Gestaltung der Arbeitsbedingungen ergeben. Ebenso gilt es zu klären, wie Unternehmen bereits erzielte Erfolge in einer nachhaltigen Gestaltung der agilen Arbeitswelt langfristig sichern und ausbauen können - und die Gefahr eines Rückfalls in alte Muster umgehen können. Dies sind aktuelle Fragen, denen in folgenden Forschungsvorhaben nachgegangen werden sollte.

\subsection{Transfermaterialien}

Andreas Boes, Katrin Gül, Tobias Kämpf, Thomas Lühr (Hrsg., 2020): Empowerment in der agilen Arbeitswelt: Analysen, Handlungsorientierungen und Erfolgsfaktoren. Freiburg: Haufe. ISBN 978-3-648-13.589-1. 
Gül, Katrin (2019): Empowerment-Index: Ein integriertes Analyse- und Gestaltungstool. In: Audi Betriebsrat, IG Metall (2019): Vorsprung durch Mitbestimmung, Broschüre.

Boes, Andreas (2019): Empowerment: Der Schlüssel zur Gestaltung der agilen Arbeitswelt. In: Audi Betriebsrat, IG Metall (2019): Vorsprung durch Mitbestimmung, Broschüre.

Boes, Andreas; Kämpf, Tobias (2019): Wie nachhaltig sind agile Arbeitsformen? In: Badura B., Ducki A., Schröder H., Klose J., Meyer M. (Hrsg.): Fehlzeiten-Report 2019, Springer, Berlin/Heidelberg, S. 193-204.

\section{Projektpartner und Aufgaben}

- Institut für Sozialwissenschaftliche Forschung e. V. München Neue Konzepte des Empowerments für Organisation, Führung und Gesundheitsförderung entwickeln

- Universität Kassel, Fachgebiet Wirtschaftsinformatik Ansätze des Empowerments für die Crowd entwickeln

- Industriegewerkschaft Metall Vorstand, Frankfurt/Main Gestaltungsoptionen für Zeitsouveränität in der digitalen Arbeitswelt bestimmen und erproben

- adrena objects ag Entwicklung von Methoden für Team-Empowerment in der agilen Softwareentwicklung

- Betriebsrat der Audi AG Ingolstadt

Neue Konzepte für eine beteiligungsorientierte Unternehmenskultur in der digitalen Arbeitswelt entwickeln

\section{Literatur}

1. Arneson H, Ekberg K (2006) Measuring empowerment in working life: A Review. In: Work, 26. Jg., H. 1, S 37-46

2. Baukrowitz A, Boes A (1996) Arbeit in der „Informationsgesellschaft“ - Einige grundsätzliche Überlegungen aus einer (fast schon) ungewohnten Perspektive. In: Schmiede, Rudi (Hrsg) Virtuelle Arbeitswelten - Arbeit, Produktion und Subjekt in der „Informationsgesellschaft". Berlin, S 129-158

3. Boes A (2005) Informatisierung. In: SOFI/IAB/ISF München/INIFES (Hrsg) Berichterstattung zur sozioökonomischen Entwicklung in Deutschland - Arbeits- und Lebensweisen. Erster Bericht. Wiesbaden, S 211-244

4. Boes A, Kämpf T, Lühr T, Marrs K (2014) Kopfarbeit in der modernen Arbeitswelt: Auf dem Weg zu einer „Industrialisierung neuen Typs“. In: Sydow J, Sadowski D, Conrad P (Hrsg) Arbeit - eine Neubestimmung. Wiesbaden, S 33-62 
5. Boes A, Kämpf T, Langes B, Lühr T (2015) Landnahme im Informationsraum. Neukonstituierung gesellschaftlicher Arbeit in der ,digitalen Gesellschaft“. In: WSI-Mitteilungen, 68. Jg., H. 2, S 77-85

6. Boes A, Kämpf T, Langes B, Ziegler A (2017) Unternehmen und die Cloud. Neue Strategien für den digitalen Umbruch und die Organisation von Arbeit? In: Arbeit, 26. Jg., H. 1, S 61-86

7. Boes A, Kämpf T, Lühr T, Ziegler A (2018a) Agilität als Chance für einen neuen Anlauf zum demokratischen Unternehmen? In: Berliner Journal für Soziologie, 28 Jg., H. 1, S 181-208. https://doi.org/10.1007/s11609-018-0367-5

8. Boes A, Kämpf T, Langes B, Lühr T (2018b) „Lean“ und ,agil“ im Büro. Neue Organisationskonzepte in der digitalen Transformation und ihre Folgen für die Angestellten. Berlin

9. Boes A, Langes B, Vogl E (2019) Die Cloud als Wegbereiter des Paradigmenwechsels zur Informationsökonomie. In: Boes A, Langes B (Hrsg) Die Cloud und der digitale Umbruch in Wirtschaft und Arbeit: Strategien Best Practices und Gestaltungsimpulse. Haufe-Lexware, Freiburg, S 115-147

10. Boudrias J-S, Gaudreau P, Laschinger H (2004) Testing the structure of psychological empowerment: does gender make a difference? Educ Psychol Measur 64. Jg., H. 5, S 861877. https://doi.org/10.1177/0013164404264840

11. Bröckling U (2003) You are not responsible for being down, but you are responsible for getting up. Über Empowerment. Leviathan, 31. Jg., H. 3, S. 323-344

12. Carless SA (2004) Does psychological Empowerment mediate the relationship between psychological climate and job satisfaction? J Bus Psychol 18. Jg., H. 4, S 405-425. https://doi. org/10.1023/B:JOBU.0000028444.77080.c5

13. Conger JA, Kanungo RN (1988) The empowerment process: Integrating theory and practice. Acad Manage Rev 13. Jg., H. 3, S 471-482. https://doi.org/10.2307/258093

14. Dörre K, Neubert J, Wolf H (1993) „New Deal“ im Betrieb? Unternehmerische Beteiligungskonzepte und ihre Wirkung auf die Austauschbeziehungen zwischen Management, Belegschaften und Interessenvertretungen. SOFI-Mitteilungen 20:15-35

15. Dörre K (1996) Die „demokratische Frage“ im Betrieb. Zu den Auswirkungen partizipativer Managementkonzepte auf die Arbeitsbeziehungen in deutschen Industrieunternehmen. SOFIMitteilungen 23:7-23

16. Dörre K (2002) Kampf um Beteiligung Arbeit, Partizipation und industrielle Beziehungen im flexiblen Kapitalismus . Wiesbaden

17. Durward D, Simmert B, Peters C, Blohm I, Leimeister JM (2019) How to empower the workforce - analyzing internal crowd work as a neo-socio-technical system. In: Hawaii International Conference on System Sciences (HICSS). Waikoloa, HI, USA

18. Hodgson D, Briand L (2013) Controlling the uncontrollable: 'Agile' teams and illusions of autonomy in creative work. Work Employ Soc 27. Jg., H. 2, S 308-325. https://doi. org/10.1177/0950017012460315

19. Kanter R M (1977) Men and women of the corporation. New York

20. Kern H, Schumann M (1984) Das Ende der Arbeitsteilung? Rationalisierung in der industriellen Produktion: Bestandsaufnahme Trendbestimmung. München

21. Langes B (2020) Cloud und der Umbruch in den Unternehmen. Empirische Fallstudien zu den Strategien von Vorreiterunternehmen. Unveröffentlichte Dissertation (im Erscheinen)

22. Laschinger H, Finegan J, Shamian J, Wilk P (2001) Impact of Structural and Psychological Empowerment on Job Strain in Nursing Work Settings: Expanding Kanter's Model. JONA J Nurs Adm 31. Jg., H. 5, S 260-272. https://doi.org/10.1097/00005110-200105000-00006

23. Levy S (1994) The empowerment tradition in American social work: a history. New York

24. Minssen H (1993) Lean production - Herausforderung für die Industriesoziologie. Arbeit, 2. Jg, H 1, S 36-52. 
25. Rappaport J (1981) In praise of paradox. A social policy of empowerment over prevention. Am J Commun Psychol 9. Jg., H. 1, S 1-25. https://doi.org/10.1007/BF00896357

26. Sauer D (2011) Von der „Humanisierung der Arbeit“ zur „Guten Arbeit“. Aus Politik und Zeitgeschichte, 61. Jg., H. 15, S 18-24

27. Simmert B, Eilers K, Peters C, Leimeister JM (2020) Agile Arbeitsorganisation fordert und fördert Empowerment - Zusammenspiel von sozialen und technischen Elementen in interner Crowd Work. In: Boes A, Gül K, Kämpf T, Lühr T (Hrsg) Empowerment in der agilen Arbeitswelt. Analysen, Handlungsorientierungen und Erfolgsfaktoren. Haufe, Freiburg, S 53-64

28. Sprafke N (2016) Kompetente Mitarbeiter und wandlungsfähige Organisationen: Zum Zusammenhang von Dynamic Capabilities, individueller Kompetenz und Empowerment. Wiesbaden

29. Spreitzer GM (1995) Psychological empowerment in the workplace: Dimensions, measurement, and validation. In: The Academy of management Journal, 38. Jg., H. 5, S 1442-1465

30. Spreitzer GM (1996) Social structural characteristics of psychological empowerment. Acad Manage J 39. Jg., H. 2, S 483-504. https://doi.org/10.2307/256789

31. Spreitzer GM (2008) Taking stock: a review of more than twenty years of research on empowerment at work. In: Cooper C, Barling J (Hrsg) Handbook of organizational behavior, 1. Jg., S 54-72

32. Thomas KW, Velthouse BA (1990) Cognitive elements of empowerment: an "interpretive" model of intrinsic task motivation. Acad Manage Rev 15. Jg., H. 4, S 666-681. https://doi. org/10.2307/258687

33. Vogl E (2018) Crowdsourcing-Plattformen als neue Marktplätze für Arbeit: Die Neuorganisation von Arbeit im Informationsraum und ihre Implikationen. Augsburg/München

34. Weibler J (2017) Empowerment - Mitarbeiter mobilisieren und binden. https://www.leadership-insiders.de/empowerment-mitarbeiter-mobilisieren-und-binden/print/. Zugegriffen: 18. Dezs 2019

35. Ziegler A, Kämpf T, Lühr T, Boes A (2020) Varieties of Empowerment - Agile Arbeitsformen in der Praxis. In: Boes A, Gül K, Kämpf T, Lühr T (Hrsg) Empowerment in der agilen Arbeitswelt. Analysen, Handlungsorientierungen und Erfolgsfaktoren. Haufe, Freiburg, S 33-52

Open Access Dieses Kapitel wird unter der Creative Commons Namensnennung 4.0 International Lizenz (http://creativecommons.org/licenses/by/4.0/deed.de) veröffentlicht, welche die Nutzung, Vervielfältigung, Bearbeitung, Verbreitung und Wiedergabe in jeglichem Medium und Format erlaubt, sofern Sie den/die ursprünglichen Autor(en) und die Quelle ordnungsgemäß nennen, einen Link zur Creative Commons Lizenz beifügen und angeben, ob Änderungen vorgenommen wurden.

Die in diesem Kapitel enthaltenen Bilder und sonstiges Drittmaterial unterliegen ebenfalls der genannten Creative Commons Lizenz, sofern sich aus der Abbildungslegende nichts anderes ergibt. Sofern das betreffende Material nicht unter der genannten Creative Commons Lizenz steht und die betreffende Handlung nicht nach gesetzlichen Vorschriften erlaubt ist, ist für die oben aufgeführten Weiterverwendungen des Materials die Einwilligung des jeweiligen Rechteinhabers einzuholen.

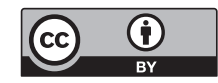

